\title{
Nucleic Acid Sequence-Based Amplification using molecular beacons for quantification of enterovirus RNA
}

\author{
Francesca Sidoti, Massimiliano Bergallo, Maria Elena Terlizzi, Sara Astegiano, \\ Cristina Costa, Rossana Cavallo \\ S.C. Virologia, U.A.O.U. San Giovanni Battista, Torino
}

Key words: NASBA, Molecular beacons, Enterovirus

Quantificazione dell'enterovirus RNA mediante Nucleic Acid Sequence-Based Amplification utilizzando sonde molecular beacons

\section{INTRODUCTION}

Enterovirus (EV) infections are very common in young children and lead to a wide spectrum of clinical presentations. Most cases are asymptomatic or mild, and usually recover without any special medication. However, especially in children, in the elderly, and in immunocompromised patients, infection can lead to death (3).

Rapid detection of enterovirus infections is essential in the management of different respiratory pathologies.

Molecular approaches have opened the way to such rapid, but also specific and sensitive, diagnostic tests. In particular, nucleic acid sequencebased amplification (NASBA) assays have been demonstrated to be more sensitive for detection of enteroviruses than RT-PCR $(2,1)$.

The aim of this study was to develop a molecular beacon-based real time NASBA assay for detection and quantification of enterovirus.

\section{METHODS}

Primers and probe oligonucleotide sequences were obtained from literature. Standards production provided different stages.

In particular, enterovirus coxsackie B4 RNA was extracted by using the automatic extractor NucliSENS easyMAG platform (bioMérieux).

Amplification reactions were carried out using the reagents from the NucliSens EasyQ Basic Kit (bioMérieux) and the the EasyQ incubator (bioMérieux).

Primers and probes were modified by adding $60 \%$ dimethyl sulfoxide (DMSO) for oligonucleotides stabilization and different primers, probes and $\mathrm{KCl}$ concentrations were tested.

In particular we used two sets of primers (200 $\mathrm{nM})$ and two probes $(30 \mathrm{nM})$ and the amplifica- tion reactions were performed at $80 \mathrm{mM} \mathrm{KCl}$. Amplification product was digested with DNase (Invitrogen) and purified using RNagent kit (Promega).

Ten fold dilutions were performed in order to obtain $10^{10}$ to $10^{\circ}$ RNA copies/reaction.

Dynamic range, sensitivity and detection limit were evaluated.

A quantity of $10^{5}$ of an internal control (U1A) was added to reaction mixture.

\section{RESULTS}

EV-RNA standard curves showed a dynamic range from $10^{8}$ to 100 copies $\left(R^{2}=0.994\right)$ and a sensitivity of 10 copies.

Limit of detection was 1 copy.

\section{CONCLUSIONS}

In conclusion NASBA assay resulted sensitive, specific and more suitable for quantification of EV. It could represent an useful tool for rapid detection of enteroviruses.

\section{REFERENCES}

1. Leone G, van Schijndel H, van Gemen B, Kramer FR, Schoen CD. Molecular beacon probes combined with amplification by NASBA enable homogeneous, realtime detection of RNA. Nucl Acids Res 1998; 26: 2150-5.

2. Patterson SS, Casper ET, Garcia-Rubio L, Smith MC, Paul JH. Increased precision of microbial RNA quantification using NASBA with an internal control. $J$ Microbiol Methods 2005; 60: 343-52.

3. Pei-Chieh W, Li-Min H, Chuan-Liang K, Tsui-Yen F, Ai-Ling C, Luan-Yin C. An outbreak of coxsackievirus A16 infection: comparison with other enteroviruses in a preschool in Taipei. $J$ Microbiol Immunol Infect 2010; 43: 271-7.

\section{Corresponding author: Francesca Sidoti}

Università di Torino, Dip. Sanità Pubblica e Microbiologia, Laboratorio di Virologia

Via Santena 9 - I0I26 Torino - Tel.: 0II 6705630 - Fax: 0 II 6705648

E-mail: francesca.sidoti@unito.it 